\title{
Effect of Strength Training on Performance of Young Competitive swimmers- A Randomized Control Trial
}

\author{
Authors \\ Roshani S.Patel ${ }^{1}$, Amit Sharma ${ }^{2}$, Ushma Prajapati ${ }^{3}$, Vaibhav Dave ${ }^{4}$, Mohindra Varma ${ }^{5}$ \\ ${ }^{1}$ Assistant Professor, Nootan College of Physiotherapy, Sankalchand Patel University, Visnagar, Gujarat, \\ India 384315 \\ ${ }^{2}$ Associate Professor, School of Physiotherapy, R.K University, Rajkot, Gujarat \\ ${ }^{3}$ Sport Physiotherapist in Amreli District Sports School, Gujarat \\ ${ }^{4}$ Sport Physiotherapist in Porbandar Sports Complex, Gujarat \\ ${ }^{5}$ Sport Physiotherapist in Rajkot, Gujarat \\ *Corresponding Author \\ Roshani S. Patel \\ Email:proshni59@gmail.com
}

\begin{abstract}
Background: Swimming, There are four styles free style, backstroke, butterfly and breaststroke. Freestyle is a top stroke in swimming. For the forward propulsion required Stroke rate and Kicking.

Aim and Objective

Aim: To Find out the effectiveness of Swimming Specific exercise regime to increase the Stroke Rate and Speed. Objective

1 To find out the effect of Core and upper or lower limb strengthening on arm Stroke in young Professional swimmers.

2 To find out the effect of Core and upper or lower limb strengthening on kicking in young Professional swimmers.

3 To compared the experimental group with the control group.

Materials and Methods

Material: Pen and Paper, Table, Chair, Height chart, Weight machine, Exercise Mat, Stop watch ,Swiss ball, DSLR video camera, Theraband.

Method: 60 male and Female swimmers were selected based on inclusion and exclusion criteria. They were given Exercises as core, upper and lower limb muscles strengthening for stroke rate and 50M kicking performance enhancement for 3 days/week for 6 weeks. Core exercises performed 1 and 2 week without Swiss ball after that 3,4,5,6 week with Swiss ball. Upper and lower limb muscles exercises performed with theraband.post data assessed after 6 Weeks, aspect stroke rate and 50M kicking assessed every 2 weeks.

Results: The data was analyzed using SPSS 21. core, upper and lower limb muscles strength Improved in Group A also stroke rate and 50M kicking speed improvement after receiving the strengthening for 6 weeks. Group B no improvement was seen.

Conclusions: There is the Significant improvement on the performance of young competitive swimmers with 6 week specific exercise regime to improve stroke rate and 50kicking peed that may be increase swimming performance in freestyle.

Keywords: Swimming, free style, stroke rate,50M kicking, strength, core, upper and lower muscles, Swiss ball, Theraband, Kickboard, DSLR video camera, State level swimmers.
\end{abstract}




\section{Introduction}

Swimming has been customary as the mainly valuable sports for an elongated time as the peoples must be able to overcome nature. ${ }^{1}$ In 1538 Nikolaus Wynmann, a German professor of languages, wrote the first book about swimming, The Swimmer or "A Dialogue on the Art of Swimming." 2 Swimming is a sport done in water through synchronized movement of the limbs and the body."3
In swimming there are four styles of freestyle, backstroke, butterfly and breaststroke. Out of four styles, freestyle is the fastest stroke in swimming races. In freestyle there is three phase, namely glide phase, pull-through phase and recovery phase. The glide phase of the stroke starts as the right hand enters the water, with the elbow slightly higher than the hand. The second phase is pull-through which is divided into 3 parts. Early pull-through, mid-pull-through and late pullthrough. ${ }^{4}$

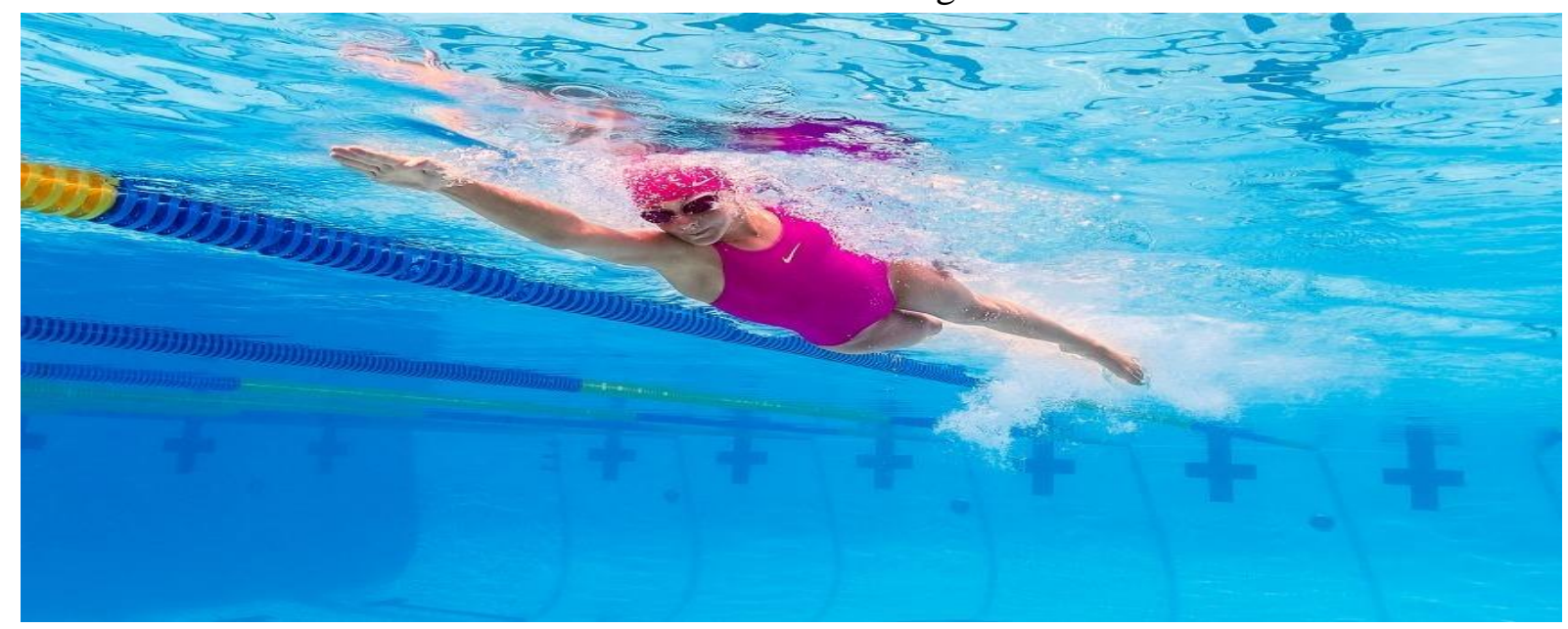

Figure 1.1 freestyle

The recovery phase has a much shorter period compared to the pull-through phase of the freestyle because of no water resistance to sluggish the movement of the arm. ${ }^{4}$

The Performance in swimming depends on the generating propel power and minimize the resistance to movement into the water. In a competitive sport of the young age swimmers, formerly associated with particular body composition. $^{5}$

The sports of swimming is very different compared to other sports because of the training is done in a prone position and utilization of equally arms and legs used for propulsion, among $90 \%$ of the propulsive force completed by the upper extremity. ${ }^{6,7}$ One more main variation, when compared to land-based sports, is to make use of core as the reference point of progress and reinforce as strong as possible to win in this sport. $^{8}$
The front crawl stroke consists of a cyclical guide of arm rotation while the legs complete a flutter kicking achievement. The arm stroke provides a greater part of the propulsion, but the legs have been contributing to the speed of movement. In Swimming with no use of the legs allow a smaller amount than maximal speeds and using only kicking action swimmers are able to push themselves through the water. The role of the flutter kicking action could be the main provider to speed in the front crawl and be able to work toward diminishes drag by keeping the body into a more streamlined position the kick also contributes by stabilizing the trunk. ${ }^{9}$

Swimming could be a recreational and extremely competitive sport which require a high level of training and performance that is why overuse of muscles lead to injuries to swimmers. To reduce the risk of injuries it must require physical attributes, both upper and lower limb strength and stroke skills. Prevalence of injuries in competitive 
swimmers is more frequency in the shoulder area $37 \%$ than knee area with $28 \%$ and foot \& ankle with $19 \%$ rate. $^{10}$

Swimming records require the swimmers to be added particularly in their training organization which has improved over the years garnering more carry from sports science. ${ }^{11}$

There is a well-built positive correlation between core muscles strength, buoyancy, and swimming performance wherever sport-specific skill is concerned, an athlete's core acts as a base of movement creation and power making most important to an improvement in the performance. $^{12,13}$

\section{Need of Study}

A strong core and upper or lower limb will make possible more energy to be transferred from the core to pull and keep the components of the stroke. If it is weak, it will spill out more energy, resulting in less kicking action. Therefore, it is most important to develop a strong core and upper or lower limb strength in swimming. However, there is little evidence proved that the core muscle strength and upper or lower limb strength would be translated and keep on improving the performance of swim time. Apart from the amount of strength a young athlete possesses in the upper and lower limbs, a weak core will finally decrease the total amount of power that can be accumulated in the extremities. So, the need of this study is to find the effect of core and upper and lower limb strengthening training on the performance of young competitive swimmers.

\section{Aim and Objective}

\section{Aim}

To find out the effectiveness of swimming specific exercise regime to increase the Stroke Rate and speed

\section{Objective}

- To find out the effect of core and upper or lower limb strengthening on arm Stroke in young Professional swimmers.
- To find out the effect of Core and upper or lower limb strengthening on kicking in young Professional swimmers.

- To compare the experimental training to the control group

\section{Hypothesis}

- Experimental Hypothesis

There is a significant effect of core strengthening combine with upper and lower strengthening exercise program when added to the routine swim training program for swimmers brings about positive changes in the performance of young competitive Swimmers.

\section{- Null Hypothesis}

There is not significant effect of core strengthening combine with upper and lower strengthening exercise program when added to the routine swim training program for swimmers brings about positive changes in the performance of young competitive Swimmers.

\section{Methodology}

\subsection{Method}

Study design: Experimental study

Study setting: Balbhavan activity hall race course, Rajkot

Sampling technique: Convenient sampling

Study population: Young competitive swimmers (male and female)

Study sample: 60

Study duration: 6 weeks

\subsection{Criteria for Selection Inclusion Criteria}

Young competitive swimmers who have participated in state-level competition both included male and female and age group 10-18

\section{Exclusion Criteria}

Subjects with any recent injury and undergoing any core training or strengthening training

\subsection{Materials Required}

Pen \& Paper

Table 
Chair

Height chart

Weight Machine

Exercise Mat

Stop Watch

Swiss Ball

DSLR Video Camera

Theraband

Kick Board

\subsection{Procedures of Data Collection}

The research was approved by the ethics committee of RK University, Rajkot. Firstly all subjects and their parents were educated about the study processes and the parents were asked to give their consent and permit their child's participation in this study. After the parent's consent, those 60 subjects were enrolled in the study. The subjects were randomly divided into 2 groups by chit method. Group-A was Experimental and Group-B was the control group. After dividing them into the groups, assessment and baseline data of the subjects were taken. Core strength measurement was done with the use of functional core strength. Upper and lower limb muscles strength were checked by the manual muscle testing, and stroke rate was observed with DSLR video camera. The 50M kicking performance was done by kicking board and checked by a stopwatch.

After the completion of all measurement Experimental group received core training and upper and lower limb muscles training along with the routine swimming training. Control group received only routine swimming training. This strengthening protocol was given for 3days/week for 6 weeks. Outcomes measures of both groups were collected after 6 weeks of training except stroke rate and 50M kicking performance, as its performance was collected every 2 weeks until 6 weeks.

\subsection{Intervention}

Core strengthening exercise included prone plank, side plank, bridging, bird dog, leg drop, dying bug along with the routine swimming training. Participants were trained for 3 times a week for six weeks. Outcome measures of both groups were collected after the end of 6 weeks of training. In 1st and 2nd week of core training, exercises were done without Swiss ball then after $3 \mathrm{rd}$, 4th, 5th an 6th weeks core training with the use of Swiss ball. ${ }^{14,15}$

\section{Week 1 and 2}

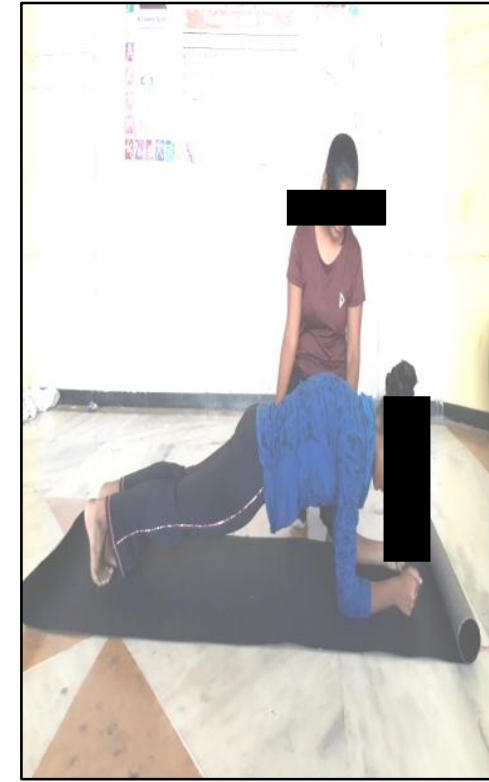

4.5.1: prone plank

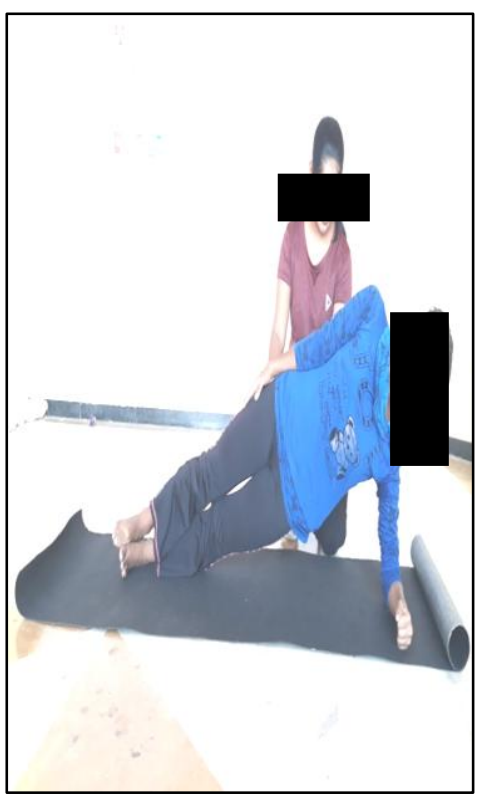

4.5.2: side plank

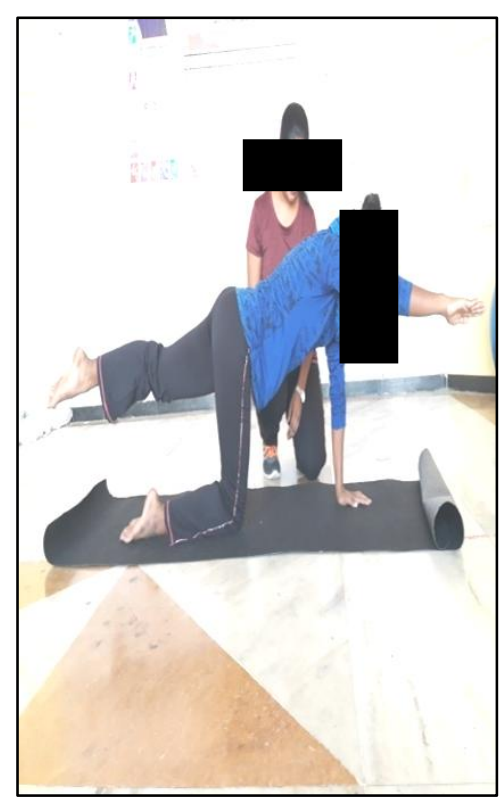

4.5.3: bird dog 


\section{JMSCR Vol||07||Issue ||05||Page 220-232||May}

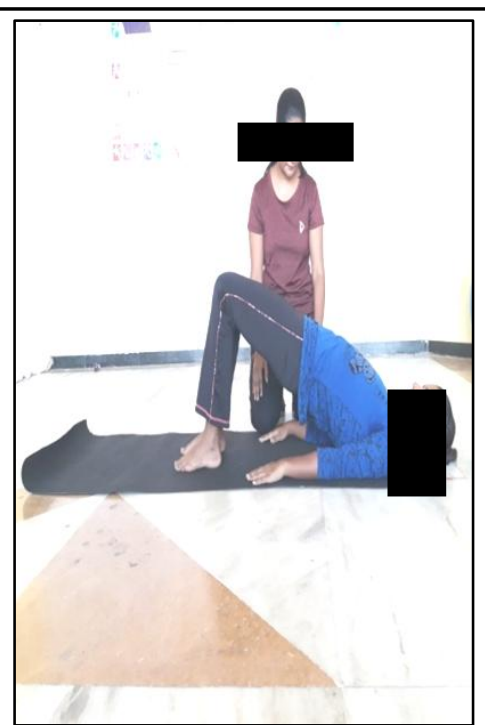

4.5.4: bridging

Week $3,4,5$ and 6

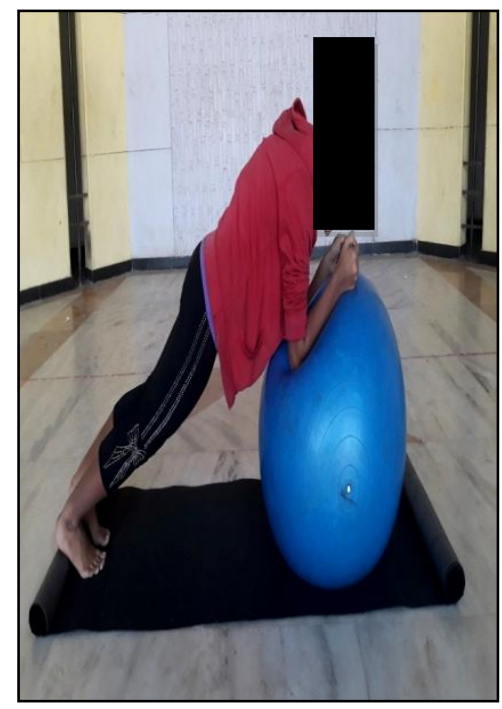

Figure 4.5.7: prone plank

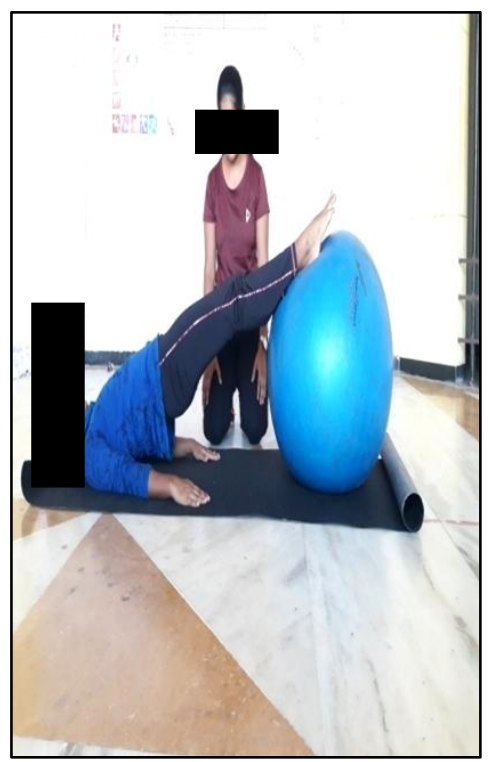

4.5.10: bringing

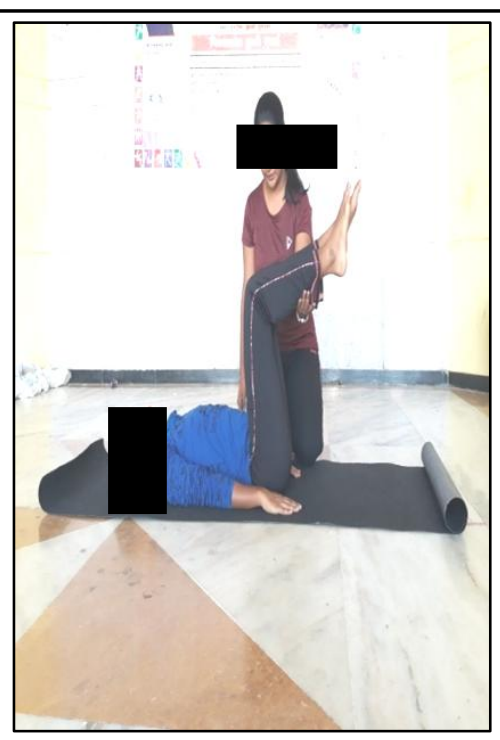

4.5.5: leg drop

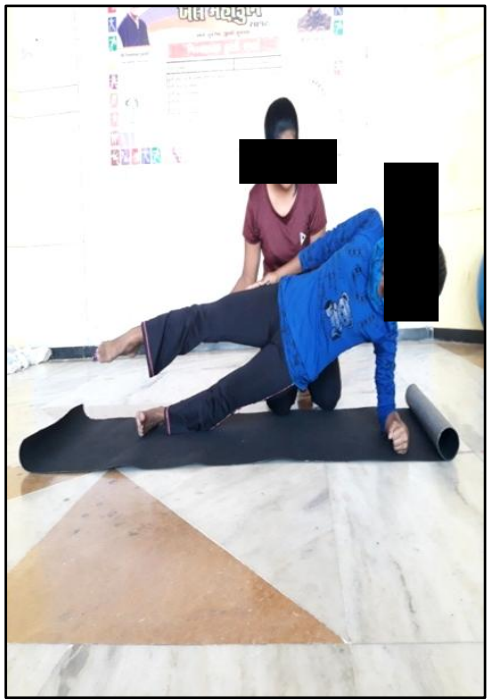

Figure 4.5.8: side plank

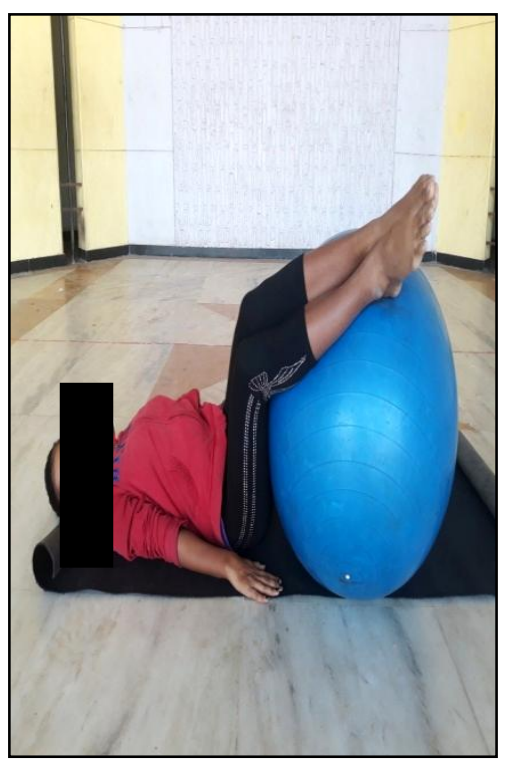

4.5.11: leg drop

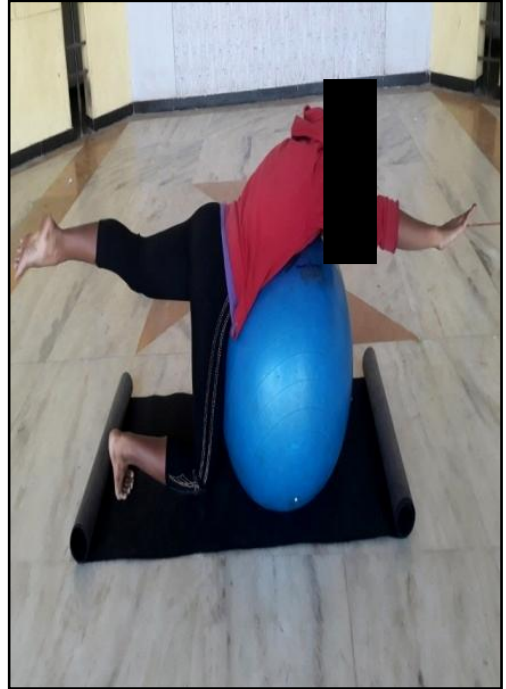

Figure 4.5.9: bird dog

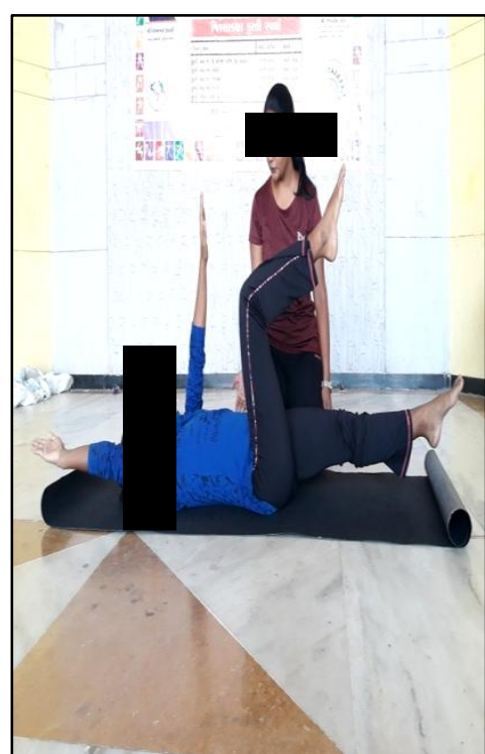

4.5.12: dying bug 


\section{JMSCR Vol||07||Issue||05||Page 220-232||May}

Upper limb strengthening exercise

The strengthening exercise of the shoulder muscles included latissimus dorsi, serratus

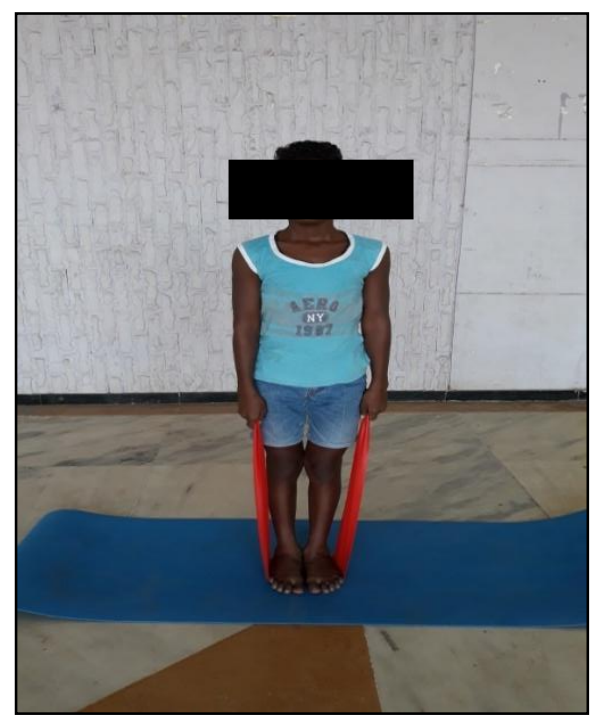

4.5.13: UT anterior, upper trapezius, with the use of theraband for 3 days a week for 6 weeks with 10 repetitions for each exercise. ${ }^{16}$

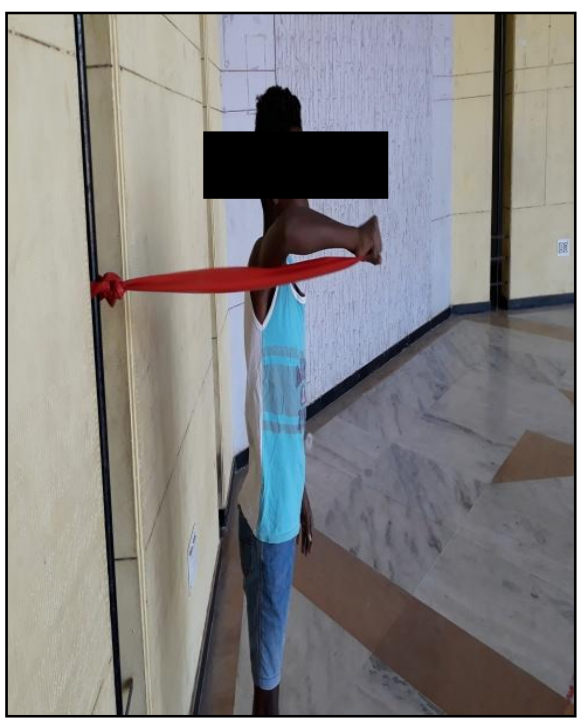

4.5.14: SA

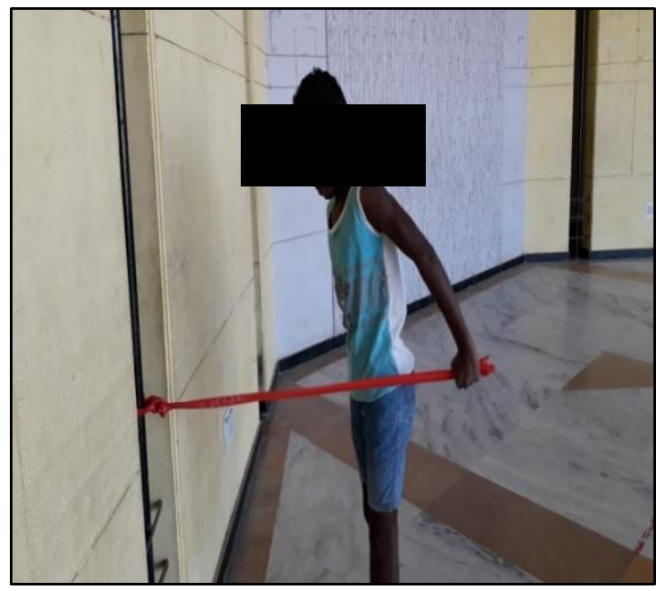

4.5.15: $\mathrm{LD}$

\section{Lower limb strengthening exercise}

The strengthening exercise of lower limb which includes hip flexors, hip extensors and ankles

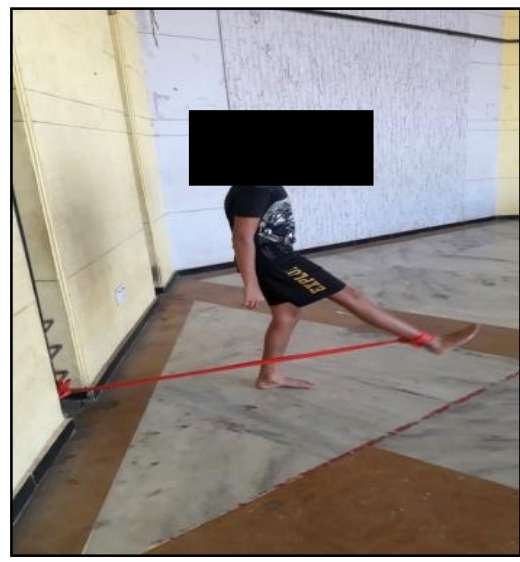

4.5.16: $\mathrm{HF}$ plantar flexors with use of theraband for 3days a week for 6 weeks with 10 repetitions for each exercise. ${ }^{16}$

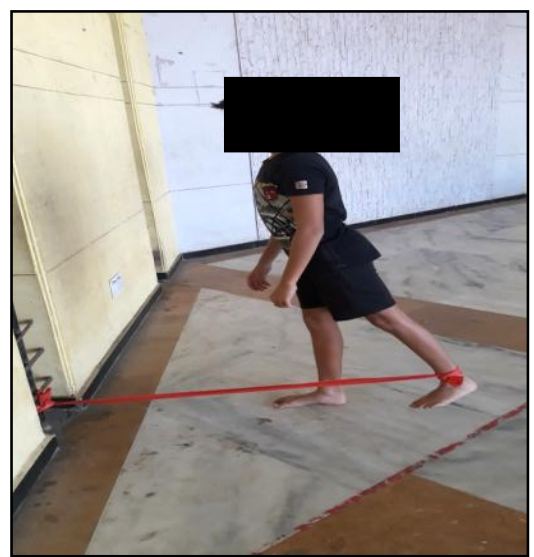

4.5.17:HE 


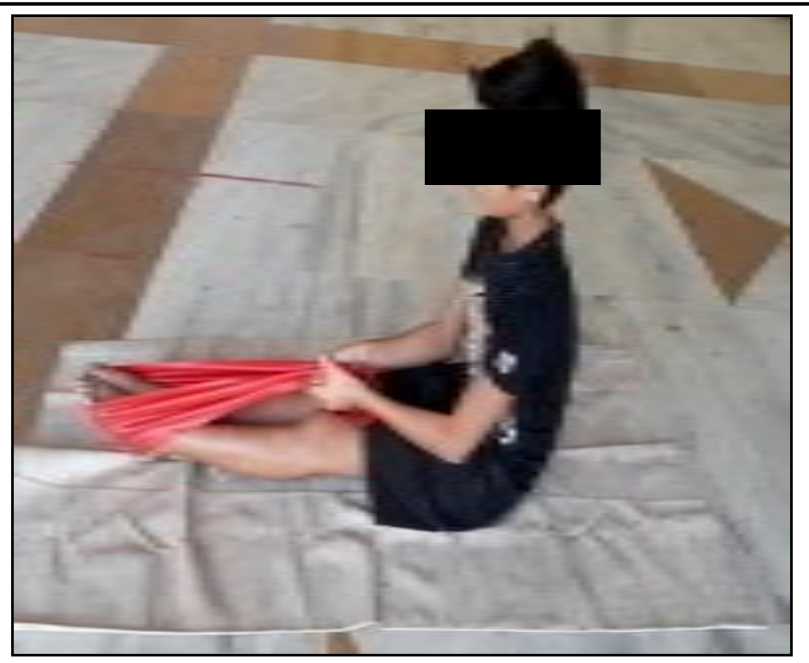

4.5.18:AP

\subsection{Outcome Measure}

Functional Core Muscle Strength Performance is a tool to assess the specific movement patterns and quality of movements in the swimming.

The Core Muscle Strength Test was used to the athlete's core strength. For this test, the subject requires a mat to support the elbows and arms and a stopwatch for the time recorded. In test included 8 stages: stage-1:hold plank position for 60 seconds,stage-2:lift right arm and hold the position for 15 seconds,stage-3:lift left arm and hold the position for 15 seconds,stage-4: lift right leg and hold the position for 15 seconds,stage-5: lift left leg and hold the position for 15 seconds,stage-6:lift right arm and left leg off the ground and hold for 15 seconds,stage-7: lift left arm and right leg off the ground and hold for 15 seconds and final stage-8: hold plank position for 30 seconds. In this, if the participated was unable to hold any of these positions then the test is to be stop. ${ }^{17,18}$

Stroke rate: Stroke Rate Done with use of DSLR Video camera and calculated by this formula: $\mathrm{SR}=$ $60 \times 3 / \mathrm{t} \mathrm{SR}$ (SR-Stroke Rate, t SR- time taken of 3 cycles). ${ }^{14}$

Kicking: The time requires completed 50M kicking performance with done of kickboard and time record by stopwatch. ${ }^{19}$

Manual muscles testing was used to check muscles strength (hip flexors, hip extensors, Ankle plantar flexors, upper trapezius and Serratus anterior and latissimus Doris). ${ }^{20}$

\section{Result}

Table 5.1: Age and Gender Distribution

\begin{tabular}{|l|c|c|c|c|c|}
\hline \multicolumn{1}{|c|}{ GROUP } & $\mathrm{N}$ & MEAN & MALE & FEMALE & P-VALUE \\
\hline EXPERIMENTAI-A & 30 & 12.47 & 16 & 14 & .882 \\
\cline { 1 - 3 } CONTROL-B & 30 & 12.47 & 11 & 19 & \\
\hline
\end{tabular}




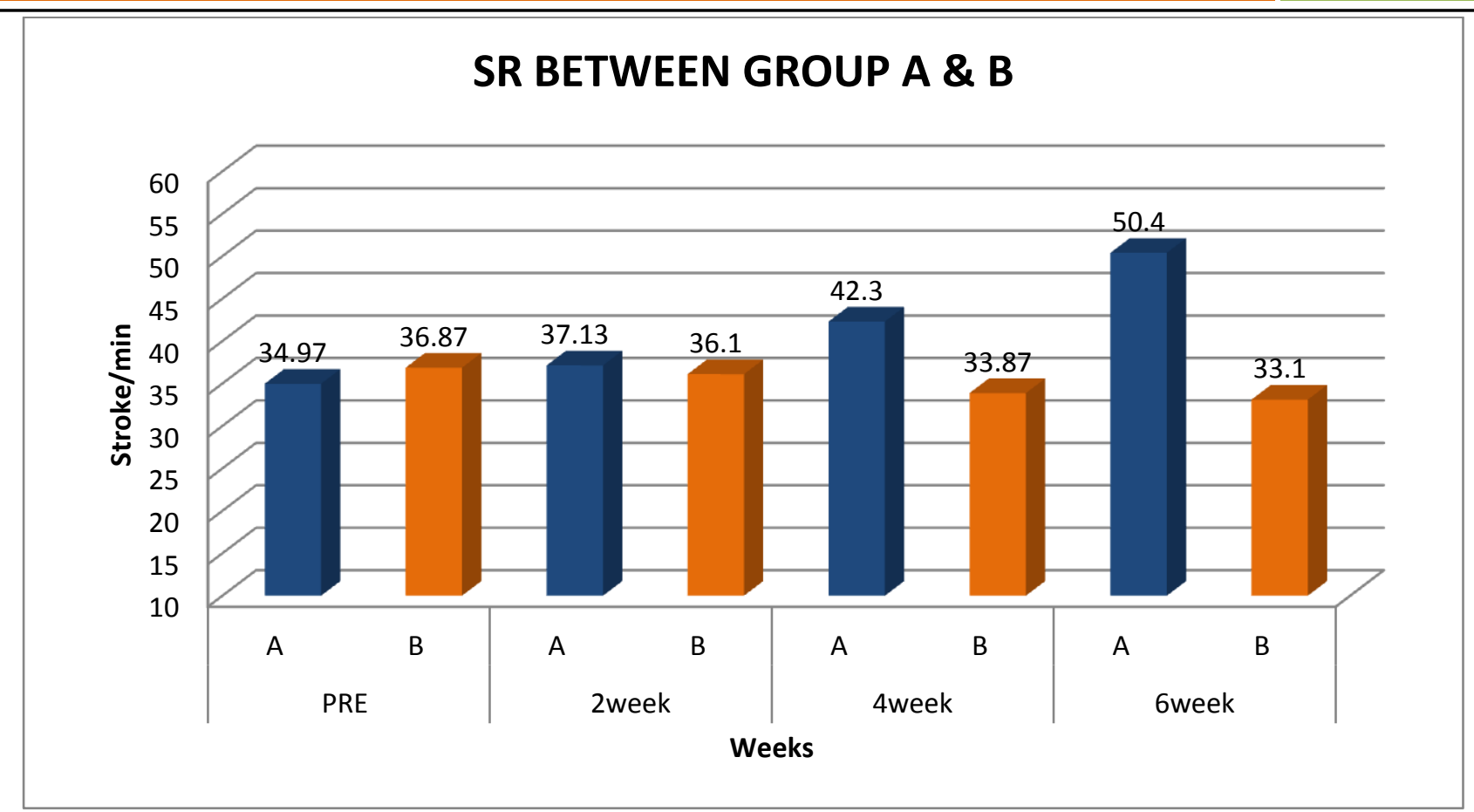

Graph 5.1: Comparison of SR Between Group A\& B

Table 5.2: Stroke Rate between Group A and B

\begin{tabular}{|l|c|c|c|}
\hline SR & GROUP & MEAN & P-VAULE \\
\hline PRE & $\mathrm{A}$ & 34.97 & .412 \\
\hline & $\mathrm{B}$ & 36.87 & \\
\hline 2WEEK & $\mathrm{A}$ & 37.13 & \multirow{2}{*}{.685} \\
\hline & $\mathrm{B}$ & 36.10 & \\
\hline 4WEEK & $\mathrm{A}$ & 42.30 & .001 \\
\hline & $\mathrm{B}$ & 33.87 & \\
\hline 6WEEK & $\mathrm{A}$ & 50.40 & \multirow{2}{*}{.000} \\
\hline & $\mathrm{B}$ & 33.10 & \\
\hline
\end{tabular}

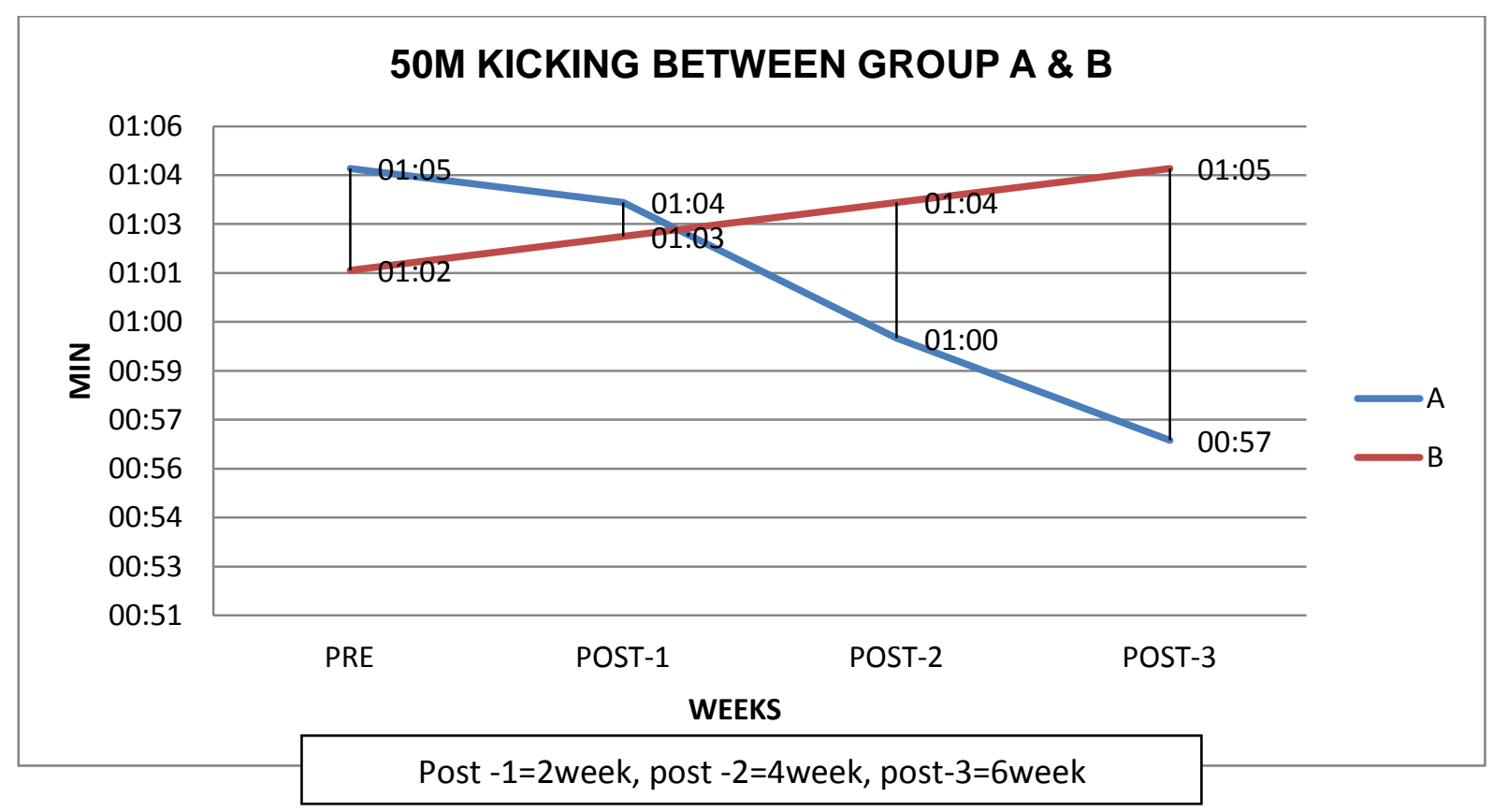

Graph 5.2: Comparison of 50m Kicking between Group A \& B 
Table 5.3:50M Kicking between Group A and B

\begin{tabular}{|l|c|c|c|}
\hline 50M KICKING & GROUP & MEAN & P-VAULE \\
\hline PRE & $\mathrm{A}$ & $1: 05$ & \multirow{2}{*}{.094} \\
\cline { 2 - 3 } & $\mathrm{B}$ & $1: 02$ & \\
\hline \multirow{2}{*}{2 WEEK } & $\mathrm{A}$ & $1: 04$ & \multirow{2}{*}{.566} \\
\cline { 2 - 3 } & $\mathrm{B}$ & $1: 03$ & \\
\hline \multirow{2}{*}{$4 \mathrm{WEEK}$} & $\mathrm{A}$ & $1: 00$ & .059 \\
\cline { 2 - 3 } & $\mathrm{B}$ & $1: 04$ & \\
\hline \multirow{2}{*}{$6 \mathrm{WEEK}$} & $\mathrm{A}$ & $0: 57$ & \multirow{2}{*}{.000} \\
\cline { 2 - 3 } & $\mathrm{B}$ & $1: 05$ & \\
\hline
\end{tabular}

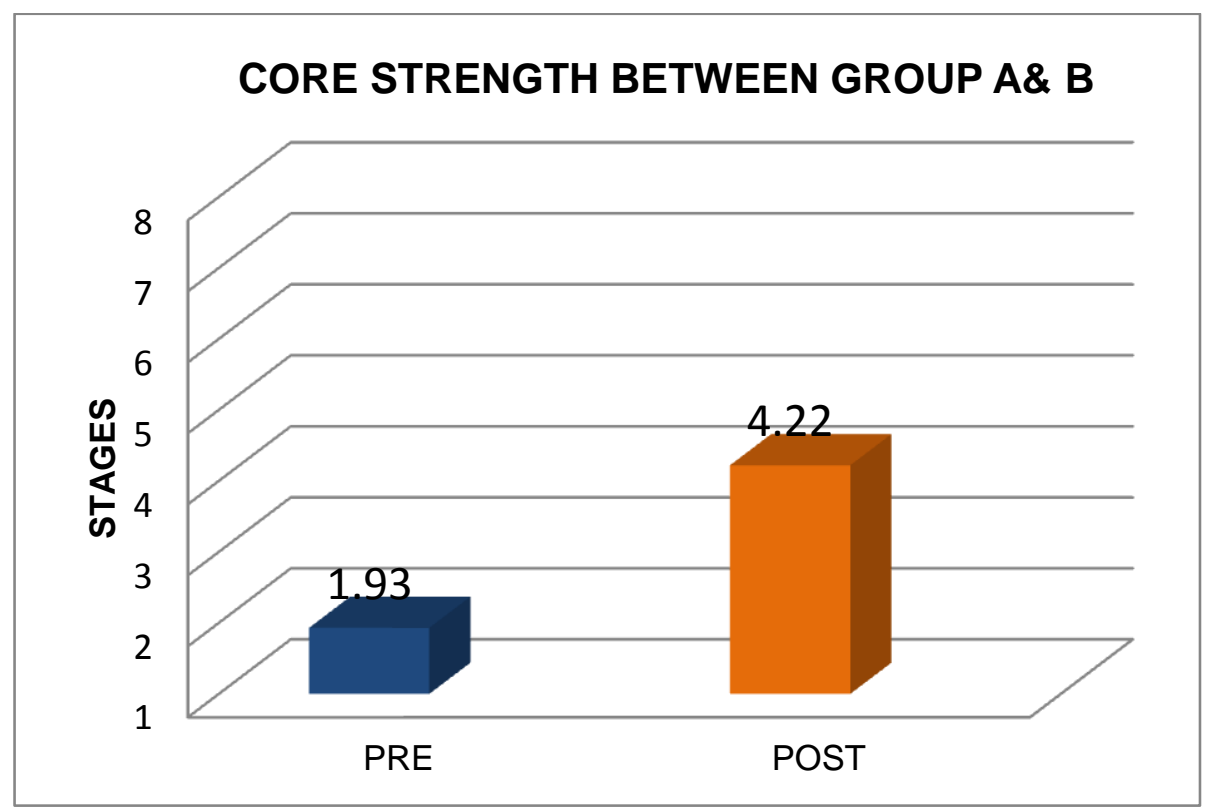

Graph 5.3: Comparison of Core Strength between Group A \& B

Table 5.4: Core Strength between Group A and B

\begin{tabular}{|l|l|l|}
\hline CORE & MEAN & P-VALUE \\
\hline PRE & 1.93 & .428 \\
\hline POST & 4.22 & .000 \\
\hline
\end{tabular}

Table 5.5: Upper Limb Muscle Strength between Group A \& B

\begin{tabular}{|l|c|c|}
\hline UL & MEAN & P-VALUE \\
\hline UT PRE & 3.80 & 1.000 \\
\hline UT POST & 4.40 & .000 \\
\hline SA PRE & 3.48 & .798 \\
\hline SA POST & 4.15 & .000 \\
\hline LD PRE & 3.85 & .282 \\
\hline LD POST & 4.40 & .000 \\
\hline
\end{tabular}

Table 5.6: Lower Limb Muscle Strength between Group A \& B

\begin{tabular}{|l|c|c|}
\hline LL & MEAN & P-VALUE \\
\hline HF PRE & 3.78 & .330 \\
\hline HF POST & 4.38 & .000 \\
\hline HE PRE & 3.83 & .492 \\
\hline HE POST & 4.40 & .000 \\
\hline AP PRE & 3.87 & .251 \\
\hline AP POST & 4.38 & .000 \\
\hline
\end{tabular}


This study included 60 young competitive swimmers. For the statistical analysis, SPSS version 21.0 was used and level of significance for All the test was kept to be 0.05

For the baselines assessment independent sample t-test was used for comparison between groups and the result shows that there was no significant difference between groups. Repeated measures ANOVA was used for SR for within groups comparison which showed that SR improved into group-A at 4 weeks $(\mathrm{p}<.021)$ and 6weeks $(\mathrm{p}<.000)$ but there was no improvement showed at 2 weeks or when comparison between groups by independent sample t-test there was showed significantly improved in group-A at 4 weeks $(p<.001)$ and 6 weeks $(p<.000)$. In group-B, there was no significant improved in SR.

Repeated measures ANOVA for 50M kicking performance within groups comparison which is showed that $50 \mathrm{M}$ kicking improved into group A at 4 weeks $(p<.053)$ and 6 weeks $(p<.000)$ but there was no improvement showed at 2 weeks or when comparison between groups by independent sample t-test there was showed significantly improved in group-A at 4 weeks $(\mathrm{p}<.059)$ and 6 weeks $(\mathrm{p}<.000)$. In group-B, there was no significant improved in $50 \mathrm{M}$ kicking Performance.

For the Core, Upper limb muscles and Lower limb muscles strength has been using a Wilcoxon signed-rank test within groups comparison and result showed that there was significantly improved in group A strength after 6 weeks of strengthening training $(\mathrm{p}<.000)$ and between group $\mathrm{A}$ and $\mathrm{B}$ comparison by mann Wheaty test and result showed that significantly improved in Group A muscles strength after 6 weeks of training $(p<.000)$.In group-B, there was no significant improved

\section{Discussion}

The aim of the study was to observe the effectiveness of swimming specific exercise regimes to increase the Stroke Rate and speed. 60 subjects were selected as per the inclusion and exclusion criteria and divided into 2 groups.
Strengthening exercises were given for 3 days/week for 6 weeks to improve swimming stroke rate and speed.

The result of the present study demonstrated that improvement in stroke rate and 50 kicking performance in group-A after strengthening protocol but there was no significant improvement in group-B. Also, there was a significant improvement in core strength, upper and lower limb muscles strength of Group A than group B after the end of the training period. So, the experimental hypothesis is accepted here.

Stroke rate is a very important factor for swimming performance which is related to muscular power and maximal swimming speed. This requires a stronger muscle to the apply propulsion force into the water and the maximum propulsion is achieved with a better stroke rate in swimming. ${ }^{21}$

According to Girolds, paul the resisted training program had a superior impact on muscle strength, swimming performance and stroke rate. ${ }^{22}$ But on another hand, Raul Arellano, peter brown said that the race distance increased there was decreased in stroke rate and velocity into female and men. ${ }^{23}$

Second most important factors in swimming were a kick which requires lower body strength that helps for forwarding propulsion along with stabilizing the trunk and streamlining the body. The streamlined position is mainly value to propulsion because the body is being prepared to move through a large amount denser medium and Drag was water resistance that is in need on body position. The more horizontal the body position is in the water, the less appearance of drag. ${ }^{24}$

However, leg kicking showed a positive correlation with speed in free stroke with a finding of Andrew D. ${ }^{25}$ Other studies Cureton, Hull that reduced the flexibility of the ankle which is the impact of speed and swimming performance. ${ }^{26}$

The current study showed that there was an improvement in the core, upper and lower limb muscle strength and this strength improved stroke rate and kicking which is most important for enhancing the swimming performance. In 
swimming, the power of the arm stroke and the speed of the leg are coordination and generate the movement.

Core stability is an important component of maximizing capable athletic function and the function produced by the kinetic chain, the coordinated sequenced activation of body segments. A core is central to all kinetic chains of upper and lower extremity function. ${ }^{27}$ The thoracolumbar fascia is an important structure that connects the upper limb (via latissimus Dorsi) to the lower limbs (via gluteus maximus) so this allows the core to be in integrated kinetic chain activity. Swimmers have to use the core as a reference point and transfer energy for the stroke and kicking. ${ }^{28}$

Many researchers have conducted that dry-land strength \& conditioning training improvement in strength. ${ }^{29}$ Jenna Sawdon-bea observed that 6weeks of dry-land exercise training was helpful for improving core strength but there was not superior shoulder strength and swimming performance. $^{30}$

The reason behind the improvement in stroke rate and kicking performance can be because of strength training there was an increase in muscle force of slow and fast twitch muscle fibers that lead to improvement in strength and produce maximal and explosive forces are required for swimming performance.

\section{Conclusion}

There is a significant improvement on the performance of young competitive swimmers with 6-week specific exercise regimes to improve stroke rate and 50kicking speed that may be increased swimming performance in freestyle.

\section{Limitations of the study}

- Small sample size

- An only Selected sample in Rajkot

Future recommendations of the study

- Carry out the study with a large sample size
- Same protocol use for other swimming styles

- Also, investigate other stroking characteristics

\section{Acknowledgement}

First I would like to thank my parents Mrs. Shilpaben patel and Mr. Satishbhai Patel for their valuable support and confidence throughout my study.

I would like to thank my guide Dr. Amit Sharma (Deputy Director, School of Physiotherapy) for the valuable guidance and advice whenever I needed.

Also I am very thankful to the Swimming Coach Mr. Bankim Joshi for supporting and allowing to carry out the data collection and for help I was whenever in need.

I would also like to show my gratitude to the Swimming Athletes who took out time for their participation in the study and helped to make my study worthy.

I express my deepest gratitude to my PG Coordinator Dr.Krupa Raithatha, who was very helpful and offered invaluable assistance, support and guidance.

I wish to express my sincere thanks to Dr. Priyanshu Rathod (Director, School of Physiotherapy) for encouragement in carrying out this project work.

I am deeply indebted to my friends and classmates, especially Dr. ushma, vaibhav, rachana, mohindra and Jaydev without whom this task would not have been possible.

I would also like to thank my younger brother Sahil Patel, to help me out with the digital work of the thesis.

\section{References}

1. Suarta, I Wayan swimming training style chest 8 times 4 sets 25 meter pool set further increase speed of 100 yards than 4 times style chest 4 sets 50 meter pool set to beginners son, ISSN : 2302-688X. Sport and Fitness Journal 2013 Volume 1, No : $45-59$. 
2. Pro.rajesh kumar. A Study on the Effect of Plyometric Exercises for Development of Speed in 50 M Back Stroke in Swimming. international. journal of physical eduction.sports and health 2014:1(1)05-07.

3. Farokie, Latiful Khobir; the effect of dry land circuit exercise model and sprint spatial training to capabilities of crawl 50 meter style speed.journal pendidikan jasmani.2016 ; volume 26 no 1.

4. Heinlein, S.A. \& Cosgarea. Biomechanical Considerations in the Competitive Swimmer's Shoulder. Sports Health: A Multidisciplinary Approach 2010; 2(6), 519-525.

5. Schneider P, Meyer F. Anthropometric and muscle strength evaluation in prepubescent and pubescent swimmer boys and girls. Rev Bras Med Esporte. 2005; 11: 4 ..

6. Aspenes, S. T. Exercise-Training Intervention Studies in Competitive Swimming. Sports Medicine2012; 42(6), 527-543.

7. Hibberd, E. E., Oyama, S. Effect of a 6Week Strengthening Program on Shoulder and Scapular-Stabilizer Strength and Scapular Kinematics in Division I Collegiate Swimmers. Journal of sport rehabilitation 2012; 21(3) 253.

8. Santana, J.C. The Essence of Stability Ball Training Companion Guide. Cranston, RI: Perform Better, 2000.; 205.

9. McCullou Volek JS, Solomon-Hill GF. Factors affecting flutter kicking speed in women who are competitive and recreational swimmers. Journal of Strength and Conditioning Research2009; 23, 21302136.

10. Dube Adiele, gundani. Prevalence of musculoskeletal injuries in male and female practicing swimming from higher school of Zimbabwe. american journal of sports science2018;6(1)8-11..

11. Toussaint H.M. Analysis of front-crawl swimming performance factors using the MAD-system: science meets practice. In:
P. Hellard, M. Sidney, C. Fauquet \& D. Lehénaff (eds.), Proceedings First international symposium sciences and practices in swimming, France: atlantica. 2006: 51-57.

12. Damsgaard R, Bencke J.Body proportions, body composition and pubertal development of children in competitive sports. Scand J Med Sci Sports 2001.

13. Kibler WB, Press J, Sciascia A. The Role of Core Stability in Athletic Function, Sports medicine 2006:36: 189-198.

14. Dr.dnyanesh patil, Dr.shivani chowdhury salian. The Effect of Core Strengthening on Performance of Young Competitive Swimmers. International Journal of Science and Research ISSN (Online): 2014; volume 3,2319-7064.

15. Imai A, Kaneoka K,Okubo. Effects of two types of trunk exercises on balance ans athletic performance in youth soccer players.int j sports phys therapy 2014;9:47.

16. Akron. Resistance band \& tubing American physical therapy association. 2012;321-2135.

17. Tom k Tong. Sport-specific endurance plank test for evaluation of global core muscle function. Physical Therapy in Sport.2014;15 58-6332.

18. Mackenzie B. 101 performance evaluation tests, in Brian Mackenzie, eds. 2005;109111.

19. Agopyan, A, Bozdogan, F.S ,Tekin, D. Acute affect of static stretching exercises onshort-distance flutter kicking time in child swimming. december. international journal of performance analysis in sport . 2012; volume12,number3.

20. Helen hislop. daniels and worthingham.' muscle testing $8^{\text {th }}$ edition.30january 2007.

21. Emmet Crowley1, Andrew J. The Impact of Resistance Training on Swimming Performance: A Systematic Review Sports Med :2017;0730-2.

22. Sebastien Girold, paul calmels, Assisted and resisted sprint training in swimming. 
Journal of strength and conditioning research,2006;20(3),547-554.

23. Raul Arellano, Peter Brown, Jane Cappaert and Richard C. Nelson. analysis of 50,100- and 200-m freestyle swimmers at the 1992 Olympic Games. journal of applied biomechanics, 1994;10,189-199.

24. Lawrence, L. The importance of the freestyle leg kick. Int Swimmer 5: 11-12, 1969.

25. ANDREW D. SORTWELL Relationship between Stroking Parameters and Leg Movement Quantity in 100 Metre Front Crawl. International Journal of Exercise Science 2011; 4(1): 22-29.

26. Cureton, TK. Mechanics and kinesiology of swimming-The crawl flutter kick. Res Q 112, 1930.

27. Putnam CA. Sequential motions of body segments in striking and throwing skills. J Biomech 1993; 26: 125-35.

28. Young JL, Herring SA, Press JM, et al. The influence of the spine on the shoulder in the throwing athlete. J Back Musculoskeletal Rehabilitation 1996; 7: 5-17.

29. Nuno M.Amaro, Daniel A. Marinho, Mario C. The effects of dry-land strength and conditioning programs in age Group Swimmers. journal of strength and conditioning research.2016:DOI:10.1519.

30. Jenna Sawdon-Bea \& Jessica Benson. the effects of a 6-week dry-land exercises program for high school swimmers. Journal of physical education and sports management.2015;vol2:1-17.

\section{List of Abbreviations}

KGS-Kilograms

$\mathrm{M}^{2}$ - Meter square

BMI-Body Mass Index

MMT-Manual Muscle Testing

50M-fifty meter

SR-Stroke rate

UL-upper limb

LL-lower limb

UT-upper trapezius

SA-serratus anterior

LD-latissimus dorsi

HF-hip flexors

HE-hip extensors

AP-ankle plantar flexors

SPSS- Statistical Package for Social Sciences 